\title{
SCIENTIFIC EXPLANATION AND METHODOLOGY OF SCIENCE
}


This page intentionally left blank 

Published by

World Scientific Publishing Co. Pte. Ltd.

5 Toh Tuck Link, Singapore 596224

USA office: 27 Warren Street, Suite 401-402, Hackensack, NJ 07601

UK office: 57 Shelton Street, Covent Garden, London WC2H 9HE

\section{British Library Cataloguing-in-Publication Data}

A catalogue record for this book is available from the British Library.

\section{SCIENTIFIC EXPLANATION AND METHODOLOGY OF SCIENCE}

Copyright (C) 2014 by World Scientific Publishing Co. Pte. Ltd.

All rights reserved. This book, or parts thereof, may not be reproduced in any form or by any means, electronic or mechanical, including photocopying, recording or any information storage and retrieval system now known or to be invented, without written permission from the publisher.

For photocopying of material in this volume, please pay a copying fee through the Copyright Clearance Center, Inc., 222 Rosewood Drive, Danvers, MA 01923, USA. In this case permission to photocopy is not required from the publisher.

ISBN 978-981-4596-63-3 


\section{PREFACE}

This volume contains the collection of selected papers from The International Conference on Scientific Explanation and Methodology of Science (SEMS 2012), held in September 17-19, 2012, in Taiyuan, Shanxi Province, China. They are revised and augmented versions of the talks presented at the Conference. SEMS 2012 is the first large-scale invitation-only international conference in philosophy of science in China. It included invited international researchers from Asia (besides China), Australia, Europe, Latin America, North America, as well as researchers from within China. Thirty-two scholars in various areas of philosophy of science give talks in the two-day conference program; and from these talks, eighteen are selected for publication in this volume. The papers represent the latest work of each researcher in his or her expertise; and as a result, this volume gives a good representation of the cutting-edge researches in diverse areas in different parts of the world, thereby achieving one of the main goals of the conference.

The conference is made possible by the generous support of the Research Centre for Philosophy of Science and Technology at Shanxi University. We would like to thank the local organizing committee for their tireless work in making sure that every aspect of the meeting is taken good care of. We would also like to thank all the invitees for their enthusiastic acceptance of our invitations and active participation in the program. We thank all the contributors of this volume for taking the time to revise or re-write their papers for the Proceedings. And finally, we want to thank the World Scientific Publishing Company for agreeing to publish the Proceedings and for supplying the copy-editing service.

Guichun Guo

Chuang Liu 
This page intentionally left blank 


\title{
ORGANIZATION
}

\author{
Co-Chairmen \\ Guichun Guo Shanxi University, China \\ Chuang Liu University of Florida, US
}

Academic Committee

Chair: Dachun Liu Renmin University of China, China

Deputy Chairs: Guichun Guo Shanxi University, China

Chuang Liu University of Florida, USA

\section{Members:}

Andreas Bartels University of Bonn, Germany

James McAllister

University of Leiden, Netherland

James R. Brown

Toronto University, Canada

Elena Castellani

University of Florence, Italy

Anjan Chakravartty

University of Notre Dame, USA

Barry Loewer

Rutgers University, USA

Alex Rosenberg

Duke University, USA

Malcolm Forster

University of Wisconsin- Madison, USA

Fan Chen

Northeastern University, China

Xinhe $\mathrm{Hu}$

Dingcheng Ren

Graduate University of Chinese Academy of Sciences, China

Graduate University of Chinese Academy of Sciences, China

Feng Lu Tsinghua University, China

Xianjing Xiao

Ce Gao

Graduate University of Chinese Academy of Sciences, China

Jie Yin

Shanxi University, China

Shanxi University, China

Ruijing Qiao

Shanxi University, China

Yidong Wei

Shanxi University, China

Peifu Zhang

Shanxi University, China 
The International Conference on Scientific Explanation and Methodology of Science Shanxi University, Taiyuan, P. R. China 2012.9

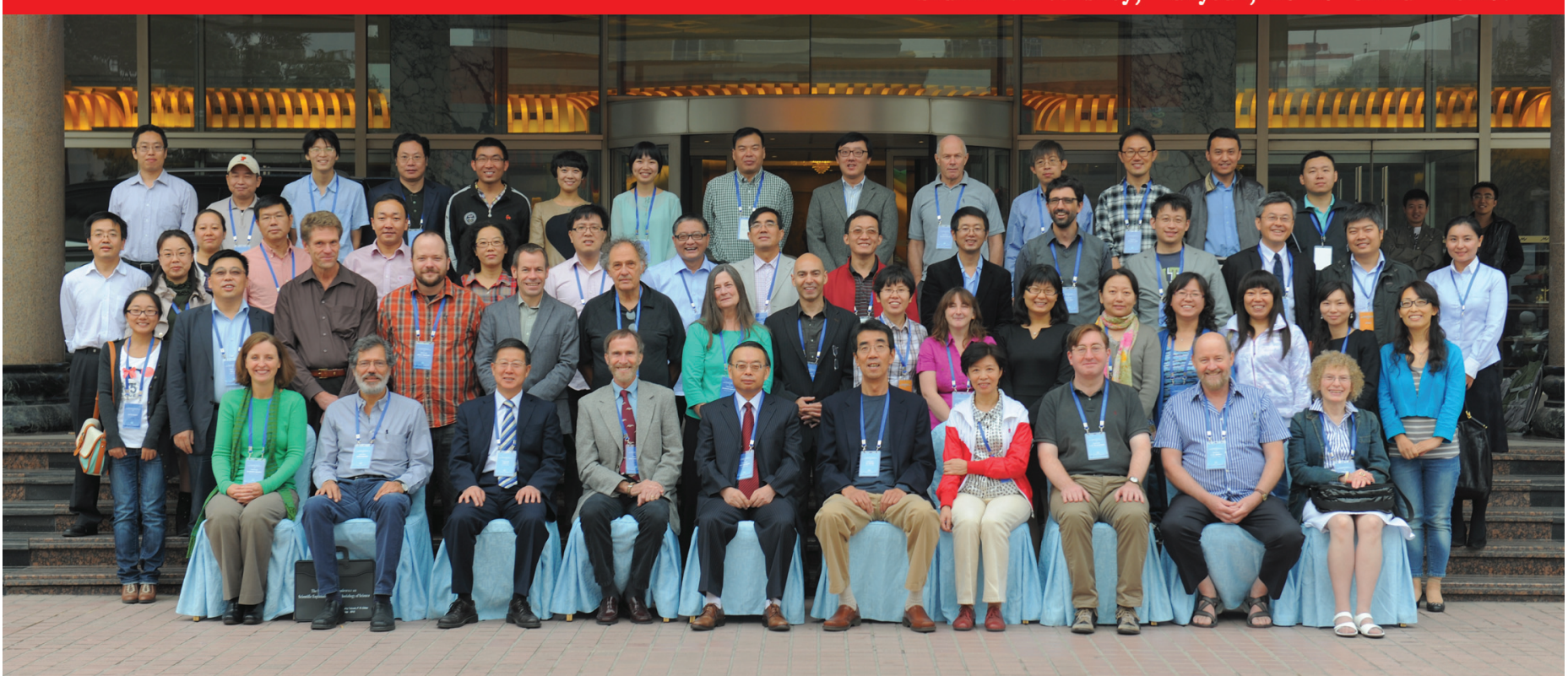




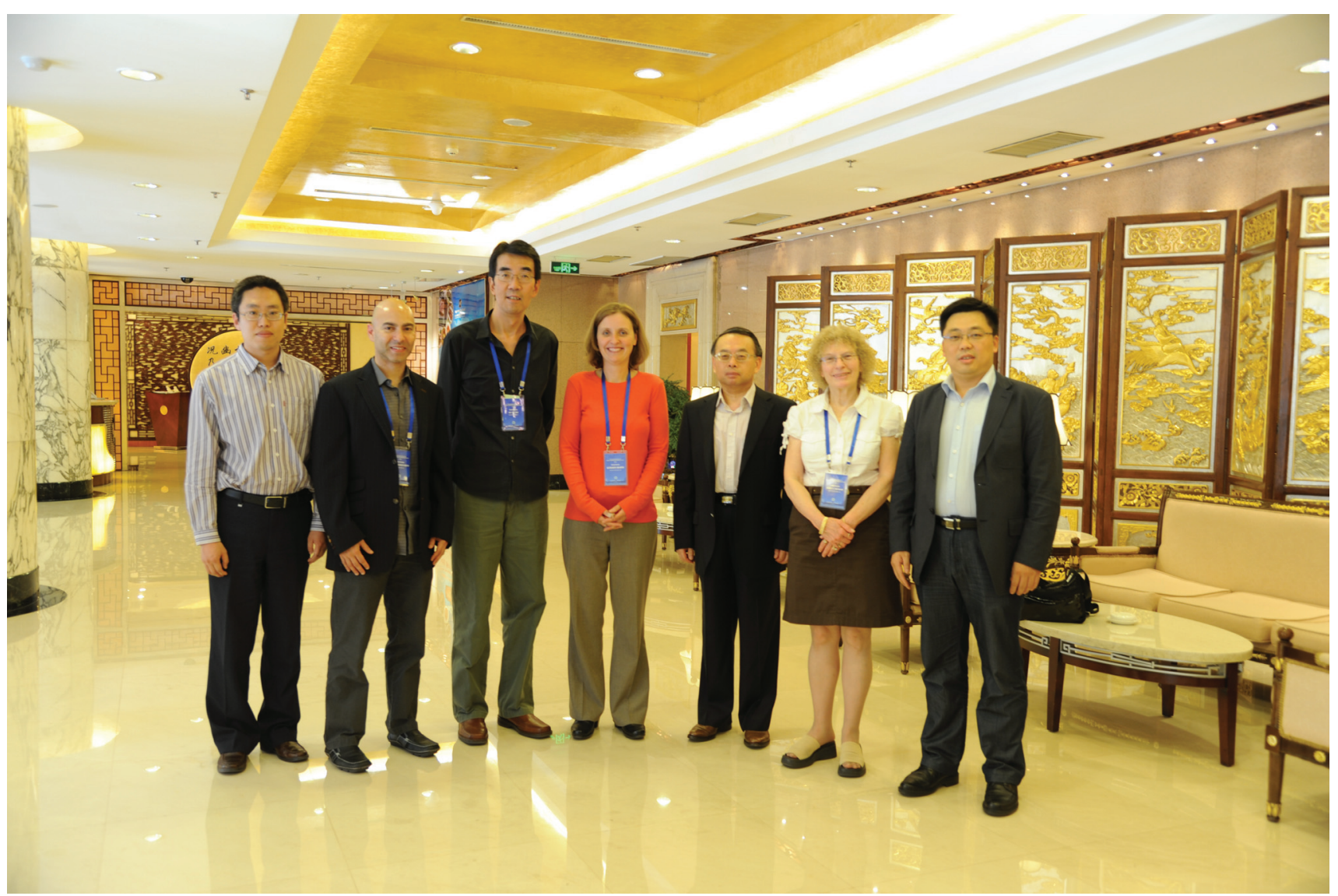

Hosts with guests from the HPS of Notre Dame.

From left: Jun An, Anjan Chakravartty, Chuang Liu, Katherine Brading, Guichun Guo, Janet Kourany, Jie Yin. 


\section{List of Participants}

\begin{tabular}{|c|c|c|c|c|}
\hline & NAME & COLLEGE & COUNTRY/REGION & E-MAIL \\
\hline 1 & Guichun Guo & Shanxi University & China & guogc@public.ty.sx.cn \\
\hline 2 & Chuang Liu & University of Florida \& Shanxi University & USA & logics@ufl.edu \\
\hline 3 & Alex Rosenberg & Duke University & USA & alexrose@duke.edu \\
\hline 4 & Andreas Bartels & University of Bonn & Germany & andreas.bartels@uni-bonn.de \\
\hline 5 & Katherine Brading & University of Notre Dame & USA & kbrading@nd.edu \\
\hline 6 & James Robert Brown & Toronto University & Canada & jrbrown@chass.utoronto.ca \\
\hline 8 & Kaiyuan Cheng & National Yang-Ming University & Taiwan & kcheng1970@gmail.com \\
\hline 9 & Sungho Choi & Kyung Hee University & South Korea & plxsc506@gmail.com \\
\hline 10 & Roman Frigg & LSE-London & UK & R.P.Frigg@lse.ac.uk \\
\hline 11 & Paul Griffiths & University of Sydney & Australia & paul.griffiths@sydney.edu.au \\
\hline 12 & Malcolm Forster & University of Wisconsin-Madison & USA & mforster@wisc.edu \\
\hline 13 & Janet A. Kourany & University of Notre Dame & USA & JKourany@nd.edu \\
\hline 15 & Sergio Martinez & The National Autonomous University of Mexico & Mexico & tepeytualco@gmail.com \\
\hline 16 & J. W. McAllister & University of Leiden & Netherlands & j.w.mcallister@phil.leidenuniv.nl \\
\hline
\end{tabular}




\begin{tabular}{|c|c|c|c|c|}
\hline & NAME & COLLEGE & COUNTRY/REGION & E-MAIL \\
\hline 17 & Ilkka Niiniluoto & University of Helsinki & Finland & niiniluo@mappi.helsinki.fi \\
\hline 18 & Kathleen Okruhlik & University of West Ontario & Canada & okruhlik@uwo.ca \\
\hline 19 & Michael Strevens & New York University & USA & strevens@nyu.edu \\
\hline 20 & Kelly Trogdon & Lingnan University & Hong Kong & old.jabo@gmail.com \\
\hline 21 & Jiji Zhang & Lingnan University & Hong Kong & jijizhang@ln.edu.hk \\
\hline 22 & Mary Leng & University of York & Canada & mary.leng@york.ac.uk \\
\hline 23 & Wei Wang & Tsinghua University & China & wangwei@tsinghua.edu.cn \\
\hline 24 & Xiang Huang & Fudan University & China & hangxiang@fudan.edu.cn \\
\hline 25 & Yingjin $\mathrm{Xu}$ & Fudan University & China & yjxu@fudan.edu.cn; \\
\hline 26 & Xiao Tan & Sun Yat-Sen University & China & tanxiao0706@gmail.com \\
\hline 27 & Qiaoying $\mathrm{Lu}$ & Sun Yat-Sen University & China & \\
\hline 28 & Xianjing Xiao & Chinese Academy of Sciences & China & Xxj201@126.com \\
\hline 29 & Liuxiang Hao & Chinese Academy of Sciences & China & \\
\hline 31 & Lian Chen & Wuhan University & China & liancheng@whu.edu.cn \\
\hline 32 & Hong Fang Li & WuHan University & China & lihongfang@whu.edu.cn \\
\hline 33 & Xiaolong Wan & Huazhong University of Science and Technology & China & hwanxl@163.com \\
\hline
\end{tabular}




\begin{tabular}{|c|c|c|c|c|}
\hline & NAME & COLLEGE & COUNTRY/REGION & E-MAIL \\
\hline 34 & Gang Chen & Huazhong University of Science and Technology & China & mgchen@hust.edu.cn \\
\hline 35 & Mingyi Chen & Huazhong University of Science and Technology & China & cmy_0223@163.com \\
\hline 36 & Hong Li & Beijing Normal University & China & lihong01@263.net \\
\hline 37 & Guolin Wu & Huanan University of Technology & China & ssglwu@scut.edu.cn \\
\hline 38 & Pei Zhang & Tianjin University of Finance and Economis & China & lizzhangpei@163.com \\
\hline 39 & Jitang Li & Soochow University & China & lijitang67@163.com \\
\hline 40 & Ge Qian & Shanghai University of Finance and Economics & China & qiange@mail.shufe.edu.cn \\
\hline 41 & Junli Guo & Shanxi University of Finance and Economics & China & \\
\hline 42 & Xiao-hong Wang & Xian Jiaotong University & China & amandawxh@sina.com \\
\hline 43 & Ce Gao & Shanxi University & China & rcpst@sxu.edu.cn \\
\hline 44 & Jie Yin & Shanxi University & China & yinjie@sxu.edu.cn \\
\hline 45 & Yidong Wei & Shanxi University & China & weiyidong@sxu.edu.cn \\
\hline 46 & Ruijin Qiao & Shanxi University & China & qrj@sxu.edu.cn \\
\hline 47 & Peifu Zhang & Shanxi University & China & zpeifu@sxu.edu.cn \\
\hline 48 & Tianping $\mathrm{He}$ & Shanxi University & China & htpsx@sxu.edu.cn \\
\hline 49 & Jun An & Shanxi University & China & anjun@sxu.edu.cn \\
\hline 50 & Shuyan Wang & Shanxi University & China & wshuyan@sxu.edu.cn \\
\hline
\end{tabular}




\begin{tabular}{|c|c|c|c|c|}
\hline & NAME & COLLEGE & COUNTRY/REGION & E-MAIL \\
\hline 51 & Shuxue Li & Shanxi University & China & lishuxue@sxu.edu.cn \\
\hline 52 & Jie Liu & Shanxi University & China & liujie@sxu.edu.cn \\
\hline 53 & Rui Cheng & Shanxi University & China & chengrui@sxu.edu.cn \\
\hline 54 & Dan Zhao & Shanxi University & China & chyuzhao@sxu.edu.cn \\
\hline 55 & Xiaohua Feng & Shanxi University & China & kinggirlss2001@sohu.com \\
\hline 56 & Bin Zhao & Shanxi University & China & sxbin-zhao@163.com \\
\hline 57 & Kaining Wang & Shanxi University & China & wangkn@sxu.edu.cn \\
\hline 58 & Yang You & Shanxi University & China & youyang@sxu.edu.cn \\
\hline 59 & Yaxin Yao & Shanxi University & China & yyx0145@sina.com \\
\hline 60 & Jianbo Guo & Shanxi University & China & gojbo@sxu.edu.cn \\
\hline 61 & Hongwu Zheng & Shanxi University & China & hwzheng@sxu.edu.cn \\
\hline 62 & Lizhen Sun & Shanxi University & China & slz@sxu.edu.cn \\
\hline 63 & Yujun $\mathrm{Xu}$ & Shanxi University & China & bianjibu@sxu.edu.cn \\
\hline 64 & Jie Yu & Shanxi University & China & bianjibu@sxu.edu.cn \\
\hline 65 & Dawei Qi & Shanxi University & China & qdw@sxu.edu.cn \\
\hline
\end{tabular}


This page intentionally left blank 


\section{CONTENTS}

Preface $\quad v$

Organization vii

List of Participants $\quad$ X

The Directedness of Time in Classical Cosmology 1 Andreas Bartels and Daniel Wohlfarth

Explanation, Special Relativity, and Presentism 16 Katherine Brading

A Muse of Fire

James Robert Brown

SSK or ESW? - The Bloor-Lynch Debate Revisited Kai-Yuan Cheng

Model Error and Ensemble Forecasting: A Cautionary Tale Seamus Bradley, Roman Frigg, Hailiang Du, and Leonard A. Smith

The Contextualist Basis of Scientific Explanation Guichun Guo

On a Bottom-Up Approach to Scientific Discovery Xiang Huang

The New Ignorance Janet A. Kourany

Models, Fiction, and Fictional Models Chuang Liu

A Contextualist Interpretation of Mathematics Jie Liu

Understanding as Integration of Heterogeneous Representations 
Approximation of Laws

Ilkka Niiniluoto

Science, Sex, and Pictures: Reflections on van Fraassen's

Use of Perspectival Representations

Kathleen Okruhlik

Bayesianism versus Confirmation

Michael Strevens

The Equivalent Transformation between Non-Truth-Function and Truth-Function Xiao-Long Wan and Ming-Yi Chen

Mechanism and Productive Event Zhu Xu

Social Science and the Bayesian Probability Explanation Model Jie Yin and Lei Zhao

Choice of Units and the Causal Markov Condition Jiji Zhang and Peter Spirtes 